\title{
Evaluation of a histocompatibility antigen related to hepatitis $B$ virus in patients with hepatocellular carcinoma in the western Brazilian Amazon
}

S.R.S. Chalub ${ }^{1}$, R.M. da Silva ${ }^{2}$, A.P.S. da Silva ${ }^{2}$, P.F. de Aquino ${ }^{3}$, A. Sadahiro ${ }^{4}$ and A.N. Kalil ${ }^{5}$

${ }^{1}$ Departamento de Cirurgia Digestiva, Universidade Federal do Amazonas, Manaus, AM, Brasil

${ }^{2}$ Departamento de Medicina, Universidade Federal do Amazonas, Manaus, AM, Brasil

${ }^{3}$ Departamento de Química, Universidade Federal do Amazonas, Manaus, AM, Brasil

${ }^{4}$ Departamento de Imunologia, Universidade Federal do Amazonas, Manaus, AM, Brasil

${ }^{5}$ Departamento de Cirurgia Digestiva,

Universidade Federal de Ciências da Saúde, Porto Alegre, RS, Brasil

Corresponding author: S.R.S. Chalub

E-mail: sidney.chalub@hotmail.com

Genet. Mol. Res. 12 (2): 1336-1346 (2013)

Received July 27, 2012

Accepted January 27, 2013

Published April 25, 2013

DOI http://dx.doi.org/10.4238/2013.April.25.5

\begin{abstract}
Hepatocellular carcinoma is an infection of variable incidence that can be caused by hepatitis B virus (HBV), which is endemic in the Amazon region. The diagnosis of HBV can be performed through the use of serum markers such as the hepatitis B surface antigen. The chronic HBV can cause mutagenesis and carcinogenesis, being the susceptibility of infection due to allele human leukocyte antigen (HLA). Thus, we evaluated the clinical, molecular and laboratory profile (histocompatibility complex) of HBV in 22 patients with hepatocellular
\end{abstract}


carcinoma in Amazonia, including 18 males and 4 females, using a blood sample for generic HLA class II. The results showed increased frequency of disease evolution in adults between 25 and 64 years old, who comprised 19 of the 22 patients studied. Most patients (16/22) presented high levels of alpha-fetoprotein and transaminases (14/22). The most common HLA alleles were DRB $1 * 04(8 / 44)$, DRB $1 * 08$ (9/44), DRB*03 (16/44), and DQB1*04 (9/44). When we compared specific phenotype frequencies of HLA-DRB ${ }^{*}$ between patients and controls, we found that patients had a significantly higher frequency of allele DRB $1 * 08$ and a significantly lower frequency of DRB $1 * 07$ and DRB $1 * 12$ compared to previous studies on Asian and Amazonian populations suggesting ethnic differences. We suggest that alleles HLADRB*08, HLA-DRB*03 and HLA-DQB1*04 may be risk factors for hepatocellular carcinoma in Amazon.

Key words: Hepatocellular carcinoma; Hepatitis B; Histocompatibility antigen

\section{INTRODUCTION}

Hepatitis B virus (HBV) infection is one of the most important human viruses. It affects approximately 300 million people worldwide and is responsible for 1-2 million annual deaths. Although most people recover from HBV infection, approximately $10 \%$ of infected individuals develop chronic hepatitis, remaining asymptomatic or developing serious liver disease such as cirrhosis or hepatocellular carcinoma (HCC) (Ferreira et al., 2006). One report has pointed out that HBV transmission occurs through blood or bodily fluids such as semen, vaginal discharge, or saliva from infected individuals, with sexual contact being the most common transmission pathway and has indicated that $15 \%$ of the population has had contact with HBV and $1 \%$ is chronically sick (Chávez et al., 2003).

Serum markers are critical for HBV diagnosis. The hepatitis B surface antigen (HBsAg) is the first marker to appear. Its persistence in serum for 6 months or more characterizes virus carriers, although it does not show complete virion replication, and an individual carrier may be asymptomatic and free of liver injury. The persistence of HBs and HBe antigens in blood circulation, antibodies against HBV core antigen (anti-HBc), and sometimes antibodies against HBs (anti-HBs) in addition to viral DNA indicates HBV chronic replication, which may be followed by progressive liver injury. A study on the prevalence of serum HBV in Brazil demonstrated that it is endemic in northern regions of the Amazonas as well as in areas of the city of Espírito Santo and the southern States of Paraná and Santa Catarina (Miranda et al., 2004).

In HCCs related to $\mathrm{HBV}, \mathrm{HBsAg}$ and anti-Hbc are usually found in serum, although $\mathrm{HBsAg}$ may be negative at low levels in some patients, maintaining positivity for anti-Hbc. The integration of viral DNA into host DNA may be the initial event affecting cellular and HBV genome alterations causing mutagenesis and carcinogenesis. The HBV DNA sequences may be identified using polymerase chain reaction (PCR) in lump tissues of HBsAg-negative patients and those with anti-Hbc and even positive serum anti-HBs (Lee, 1997). The application of molecular biology techniques has allowed notable achievements in HBV research (Ferreira, 2000). 
Acute or chronic illness is conditioned by the mechanism of immune response regulated by the human leukocyte antigen (HLA) molecule. The chronic susceptibility of infection is due to allele HLA-A0206 in the Taiwanese population, B35 in the Chinese, B18, B35, B40, and $\mathrm{Cw} 3$ in Russians, and B44-Cw 0501 in white Americans. HLA-B8 in white Europeans is associated with the absence of response to HBV vaccine. In the Chinese population, HLADR3 is associated with vertical transmission (Singh et al., 2007).

HBV infection clearance was specifically associated with HLA-DR13 in several ethnic groups. In Gambia, the HLA-DRB1-1302 allele is associated with protection against persistent HBV infection. A European study has confirmed that alleles HLA-DRB1-1302 and HLA-DRB-1301 also show resistance to chronic infection in whites (Carrilho et al., 2010). In a study of 91 American adults and Africans, 60 presented persistent $\mathrm{HBV}$ infection related to the alleles HLA-DQA1 0501 and HLA-DQB1 0301. Another study has shown a strong association of HLA-DRB1 0301, HLA-DQA1 0501, and HLA-DQB1 0301 with susceptibility to chronic hepatitis B (Kummee et al., 2007). We evaluated the serum profile and identified the antigen of histocompatibility (HLA class II), aiming to verify risk factors for HCC in a population infected by hepatitis in a health clinic in the western Brazilian Amazonia.

\section{MATERIAL AND METHODS}

\section{Study model}

This study was approved by the Ethics Council for Research of Human Beings of the Federal University of Amazon (MEMO - No. 0132.0.115.000-10-CAAE). This casecontrol study included patients of both genders, older than 13 years, who were carriers of HBV and HCC and were seen at the Oncology Control Foundation Center of the Amazonas State (FCECON) between August 2010 and July 2011. The controls were patients of both genders, aged 13 years and older, who were non-carriers of HBV and HCC and registered in the database of the Laboratory of Immunology of the Federal University of Amazonas, and who performed HLA tests.

\section{Study population}

Patients were included in this study according to the following criteria: they were hepatitis B patients with HCC, had been seen at the Cancer Foundation during the study period, were older than 13 years, had positive serum for HBV evaluated through specific markers (HBsAg, anti-HBs, total anti-HBc, $\mathrm{HBeAg}$, and anti-HBe), and had confirmed presence of $\mathrm{HCC}$ on imaging tests including ultrasound, tomography, or magnetic nuclear resonance and alpha-fetoprotein dosage. All patients signed informed consent forms after the medical procedures were explained.

HBV and HCC patients attended the ambulatory unit of the FCECON on Tuesdays beginning at 10:00 am. The patients completed a questionnaire, and $10 \mathrm{~mL}$ venous blood was drawn by a phlebotomist. A total of $5 \mathrm{~mL}$ blood was collected in a tube with anticoagulant and $5 \mathrm{~mL}$ was collected in a tube without anticoagulant. All samples were kept in a polystyrene container with ice gel at the Immunology Laboratory of the Federal University of Amazonas and analyzed for HLA using the methods described below. 


\section{DNA extraction}

DNA extraction from venous blood was carried out with a rapid technique using salts of tetraethylammonium bromide [dodecyltrimethylammonium bromide (DTAB)/cetyltrimethylammonium bromide (CTAB)] described by Gustincich et al. (1991) with the following adaptations. The leukocyte layer, the "buffy coat", was obtained from centrifuged blood at $250 \mathrm{~g}$ for $10 \mathrm{~min}$ after mixing with an equal volume of lysis buffer [DTAB $12 \%$ (12\% DTAB, $25 \mathrm{M} \mathrm{NaCl}_{2}, 150 \mathrm{mM}$ Tris $150, \mathrm{pH} 8.6$, and $75 \mathrm{mM}$ ethylenediaminetetraacetic acid)] and incubated for $10 \mathrm{~min}$ at $68^{\circ} \mathrm{C}$. Two volumes of chloroform were added after strong agitation. After centrifugation at $10,000 \mathrm{~g}$ for $2 \mathrm{~min}$, the supernatant was recovered and added to two volumes of CTAB $0.5 \%(5 \% \mathrm{CTAB}, 0.4 \mathrm{M} \mathrm{NaCl})$ and then homogenized until DNA/ CTAB precipitated.

After additional centrifugation at $10,000 \mathrm{~g}$ for $2 \mathrm{~min}$, the precipitation was suspended in $300 \mu \mathrm{L} 1.2 \mathrm{M} \mathrm{NaCl}$ and $750 \mu \mathrm{L}$ absolute ethanol and centrifuged for $2 \mathrm{~min}$ at $16,000 \mathrm{~g}$. Additional washings and centrifugations were carried out with $70 \%$ ethanol. The supernatant was discharged, and the precipitation was dissolved in $200 \mu \mathrm{L} \mathrm{H}_{2} \mathrm{O}$.

\section{Generic HLA class II (DRB1, DRB3, DRB4, DRB5, and DQB1)}

After DNA extraction and normalization, the samples were typed for generic HLA class II using a micro-sequence-specific primer (SSP) plate from One Lambda ${ }^{\circledR}$. The SSP technique described by Olerup and Zetterquist (1992) was used with modification. Samples from three patients in triplicate were mixed with Taq polymerase and PCR reagents before loading onto a One-Lambda 96-well DMIX plate according to manufacturer instructions.

The amplification of a 150 -bp product was checked with $1.5 \%$ agarose gel electrophoresis under ultraviolet light. The final HLA allele typing analysis was performed using DNALMT version 3.81 (One Lambda).

\section{Analysis and data interpretation}

The variables were tabulated in Excel 2007 and the statistical analyses were carried out with MINITAB version 14.1. The frequencies of the HLA class II antigens were recorded in tables of frequency distributions. The relationships between the histocompatibility HLA class II antigen and HBV disease evolution with respect to anti-HBc and gender were determined with chi-square and Fisher exact tests (when the chi-square test was unavailable) considering a level of $5 \%$ of significance in all verifications.

\section{RESULTS}

The study population was $81.8 \%$ men and $18.2 \%$ women. All patients were in an advanced stage of disease, with some displaying cirrhosis. The majority of patients were aged 20-45 years, whereas the minority was older than 65 years. A follow-up of the study population verified that approximately $73 \%$ became sick and $14 \%$ died, which constituted $87 \%$ of the sample. Only $14 \%$ were cured (Figure 1). Most (86.4\%) presented reactivity to the anti-HBc marker, whereas only $13.6 \%$ showed no reactivity. 


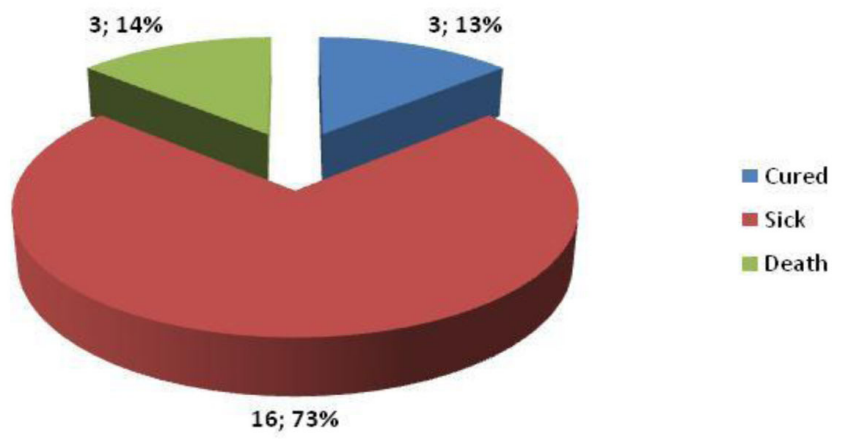

Figure 1. Distribution changes in the population studied.

DNA was extracted from 22 blood samples, quantified, and submitted to generic class II complex of histocompatibility typing (HLA-DRB1*, HLA-DRB3*, HLA-DRB4*, HLADRB5*, and HLA-DBQ1*). Because each individual represents two alleles, 44 alleles were analyzed. As shown in Figure 2, HLA-DRB $1 * 08$ prevailed, followed by HLA-DRB1*04; both composed approximately $39 \%$ of the evaluated samples. No results were found for HLADRB1*10 and HLA-DRB1*09.

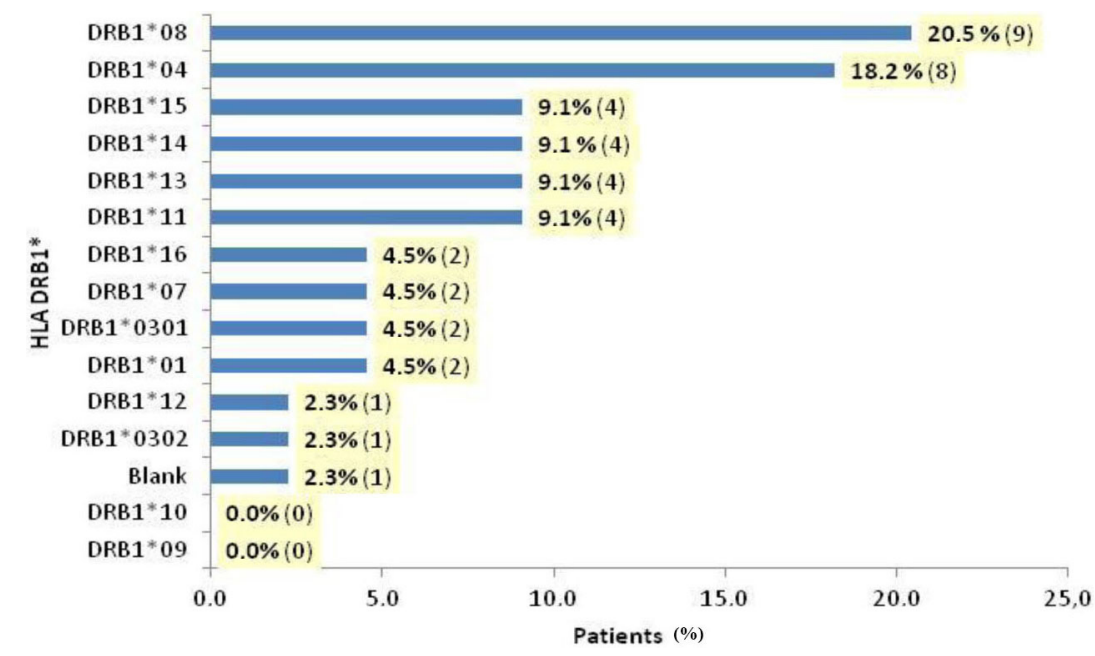

Figure 2. Distribution of human leukocyte antigen (HLA)-DRB1* generic among patients with hepatitis B and hepatocellular carcinoma.

Considering the phenotype frequencies for specificities of HLA-DRB1* (Table 1) between patients and controls, patients presented significantly lower HLA-DRB $1 * 07$ frequency and significantly higher HLA-DRB $1 * 08$ frequency compared with those in controls $(\mathrm{P}<0.05)$. No evidence of a significant association was found between HLA-DRB1* specificities and anti-HBc serum markers in the sample studied (Table 2; $\mathrm{P}>0.05$ ). No evidence of significance was found between the HLA-DRB1* specificities and disease evolution in the sample studied $(\mathrm{P}>0.05)$. 
Table 1. Comparison between the phenotypic frequencies of human leukocyte antigen (HLA)-DRB1* alleles among patients infected with the hepatitis B virus and the group of uninfected patients (control).

\begin{tabular}{lccc}
\hline HLA-DRB1* & Pacients $[(\mathrm{N}=44) \%]$ & Controls $[(\mathrm{N}=44) \%]$ & P value \\
\hline Blank & 2.3 & 13.6 & 0.05 \\
DRB1*01 & 4.5 & 4.5 & 0.62 \\
DRB1*04 & 18.2 & 13.6 & 0.77 \\
DRB1*07 & 4.5 & 20.5 & 0.02 \\
DRB1*08 & 20.5 & 13.6 & 0.02 \\
DRB1*09 & 0.0 & 4.5 & 0.25 \\
DRB1*10 & 0.0 & 0.0 & -0.06 \\
DRB1*11 & 9.1 & 0.0 & 0.50 \\
DRB1*12 & 2.3 & 0.0 & 0.73 \\
DRB1*13 & 9.1 & 13.6 & 0.35 \\
DRB1*14 & 9.1 & 2.3 & 0.61 \\
DRB1*15 & 9.1 & 6.3 & 0.50 \\
DRB1*16 & 4.5 & 4.5 & 0.69 \\
DRB1*0301 & 4.5 & 0.0 & 0.50 \\
DRB1*0302 & 2.3 & &
\end{tabular}

Table 2. Distribution of human leukocyte antigen (HLA)-DRB1* generic towards the scoring results for anti$\mathrm{HBc}$ in patients with hepatocellular carcinoma.

\begin{tabular}{|c|c|c|c|c|c|c|}
\hline \multirow[t]{2}{*}{ Results of HLA-DRB1* } & \multicolumn{4}{|c|}{ Anti-HBc } & \multirow[t]{2}{*}{ Total } & \multirow[t]{2}{*}{$P$ value } \\
\hline & Positive & $\%$ & Negative & $\%$ & & \\
\hline Blank & 1 & 100.0 & 0 & 0.0 & 1 & 0.86 \\
\hline DRB1*01 & 2 & 100.0 & 0 & 0.0 & 2 & 0.74 \\
\hline DRB $1 * 0301$ & 2 & 100.0 & 0 & 0.0 & 2 & 0.74 \\
\hline DRB $1 * 0302$ & 1 & 100.0 & 0 & 0.0 & 1 & 0.86 \\
\hline DRB1*04 & 7 & 87.5 & 1 & 12.5 & 8 & 0.70 \\
\hline $\mathrm{DRB} 1 * 05$ & 1 & 100.0 & 0 & 0.0 & 1 & 0.86 \\
\hline DRB1*07 & 2 & 100.0 & 0 & 0.0 & 2 & 0.74 \\
\hline $\mathrm{DRB} 1 * 08$ & 7 & 77.8 & 2 & 22.2 & 9 & 0.35 \\
\hline $\mathrm{DRB} 1 * 11$ & 3 & 75.0 & 1 & 25.0 & 4 & 0.45 \\
\hline $\mathrm{DRB} 1 * 12$ & 0 & 0.0 & 1 & 100.0 & 1 & 0.13 \\
\hline $\mathrm{DRB} 1 * 13$ & 4 & 100.0 & 0 & 0.0 & 4 & 0.54 \\
\hline $\mathrm{DRB} 1 * 14$ & 3 & 75.0 & 1 & 25.0 & 4 & 0.45 \\
\hline DRB $1 * 15$ & 3 & 100.0 & 0 & 0.0 & 3 & 0.63 \\
\hline $\mathrm{DRB} 1 * 16$ & 2 & 100.0 & 0 & 0.0 & 2 & 0.74 \\
\hline Total & 38 & 86.4 & 6 & 13.6 & 44 & \\
\hline
\end{tabular}

We noticed that the phenotype frequency was significantly lower in male patients compared to that in females for the HLA-DRB1*14 allele $(\mathrm{P}<0.05)$, as presented in Table 3.

Table 3. Distribution of human leukocyte antigen (HLA)-DRB1* generic in relation to gender of patients with hepatocellular carcinoma.

\begin{tabular}{|c|c|c|c|c|c|c|}
\hline Results of HLA-DRB1* & Male & $\%$ & Female & $\%$ & Total & $P$ value \\
\hline Blank & 1 & 100.0 & 0 & 0.0 & 1 & 0.82 \\
\hline $\mathrm{DRB} 1 * 01$ & 2 & 100.0 & 0 & 0.0 & 2 & 0.67 \\
\hline DRB $1 * 0301$ & 2 & 100.0 & 0 & 0.0 & 2 & 0.67 \\
\hline $\mathrm{DRB} 1 * 0302$ & 1 & 100.0 & 0 & 0.0 & 1 & 0.82 \\
\hline $\mathrm{DRB} 1 * 04$ & 6 & 75.0 & 2 & 25.0 & 8 & 0.45 \\
\hline DRB $1 * 05$ & 1 & 100.0 & 0 & 0.0 & 1 & 0.82 \\
\hline DRB1*07 & 2 & 100.0 & 0 & 0.0 & 2 & 0.67 \\
\hline $\mathrm{DRB} 1 * 08$ & 7 & 77.8 & 2 & 22.2 & 9 & 0.53 \\
\hline DRB $1 * 11$ & 4 & 100.0 & 0 & 0.0 & 4 & 0.43 \\
\hline $\mathrm{DRB} 1 * 12$ & 1 & 100.0 & 0 & 0.0 & 1 & 0.82 \\
\hline $\mathrm{DRB} 1 * 13$ & 4 & 100.0 & 0 & 0.0 & 4 & 0.43 \\
\hline $\mathrm{DRB} 1 * 14$ & 1 & 25.0 & 3 & 75.0 & 4 & 0.02 \\
\hline DRB $1 * 15$ & 2 & 66.7 & 1 & 33.3 & 3 & 0.46 \\
\hline $\mathrm{DRB} 1 * 16$ & 2 & 100.0 & 0 & 0.0 & 2 & 0.67 \\
\hline Total & 36 & 81.8 & 8 & 18.2 & 44 & \\
\hline
\end{tabular}


HLA-DRB3* was predominant for these specificities, whereas HLA-DRB5* was less frequent. Considering phenotype frequencies for the HLA-DRB3*, HLA-DRB4*, and HLADRB5* specificities, no evidence of significant differences between allele frequencies were found between patients and controls $(\mathrm{P}>0.05)$. In addition, we found no evidence of an association between HLA-DRB3*, HLA-DRB4*, and HLA-DRB5* specificities and the anti-HBc serum marker in the sample studied $(\mathrm{P}>0.05)$. As shown in Table 4 , no significant association was found between HLA-DRB3*, HLA-DRB4*, and HLA-DRB5* specificities and the disease evolution in the sample studied $(\mathrm{P}>0.05)$.

\begin{tabular}{|c|c|c|c|c|c|c|}
\hline Results of HLA-DRB3*, -DRB4*, -DRB5* & Healthy & $\%$ & Sick & $\%$ & Total & $P$ value \\
\hline Blank & 1 & 8.3 & 11 & 91.7 & 12 & 0.47 \\
\hline DRB3* & 3 & 18.8 & 13 & 81.3 & 16 & 0.38 \\
\hline DRB4* & 2 & 20.0 & 8 & 80.0 & 10 & 0.41 \\
\hline DRB5* & 0 & 0.0 & 6 & 100.0 & 6 & 0.39 \\
\hline Total & 6 & 13.6 & 38 & 86.4 & 44 & \\
\hline
\end{tabular}

In relation to gender, no significant difference was found between the HLA-DRB*3, HLA-DRB4*, and HLA-DRB5* alleles ( $\mathrm{P}>0.05)$, as shown in Table 5.

\begin{tabular}{|c|c|c|c|c|c|c|}
\hline Results of HLA-DRB3*, -DRB4*, -DRB5* & Male & $\%$ & Female & $\%$ & Total & $P$ value \\
\hline Blank & 10 & 83.3 & 2 & 16.7 & 12 & 0.62 \\
\hline DRB3* & 13 & 81.3 & 3 & 18.8 & 16 & 0.62 \\
\hline DRB4* & 8 & 80.0 & 2 & 20.0 & 10 & 0.59 \\
\hline DRB5* & 5 & 0.0 & 1 & 16.7 & 6 & 0.70 \\
\hline Total & 36 & 81.8 & 8 & 18.2 & 44 & \\
\hline
\end{tabular}

Figure 3 shows that HLA-DBQ $1 * 04(20.5 \%)$ prevailed, followed by HLA-DRB $1 * 0301$ $(18.2 \%)$, and HLA-DBQ $1 * 06(18.2 \%)$, comprising approximately $57 \%$ of the study.

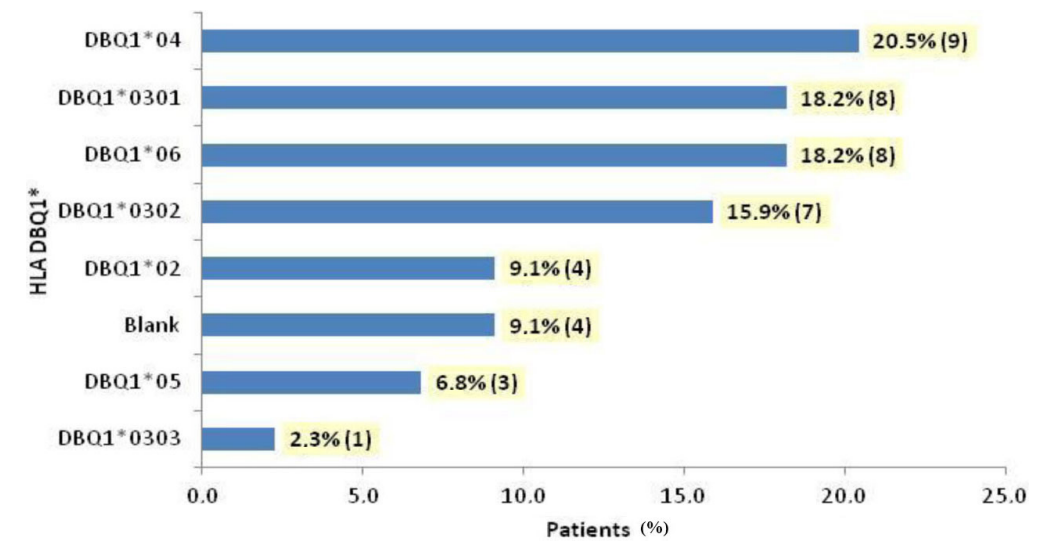

Figure 3. Distribution of human leukocyte antigen (HLA)-DBQ1* generic among patients with hepatitis B and hepatocellular carcinoma. 
The phenotype frequencies for this specificity, shown in Table 6, demonstrate that patients had a significantly lower HLA-DBQ $1 * 02$ frequency compared with that in controls $(\mathrm{P}<0.05)$. No evidence of a significant difference between patients and controls was found for the other allele frequencies.

\begin{tabular}{|c|c|c|c|}
\hline HLA-DRB1* & Patients $[(\mathrm{N}=44) \%]$ & Controls $[(\mathrm{N}=44) \%]$ & $P$ value \\
\hline Blank & 9.1 & 11.4 & 0.01 \\
\hline DBQ $1 * 02$ & 9.1 & 27.3 & 0.02 \\
\hline DBQ $1 * 04$ & 20.5 & 6.8 & 0.06 \\
\hline DBQ $1 * 05$ & 6.8 & 4.6 & 0.50 \\
\hline DBQ $1 * 06$ & 18.2 & 20.5 & 0.79 \\
\hline DBQ1*0301 & 18.2 & 11.4 & 0.54 \\
\hline DBQ1*0302 & 15.9 & 15.9 & 0.78 \\
\hline DBQ1*0303 & 2.3 & 2.3 & 0.75 \\
\hline
\end{tabular}

\section{DISCUSSION}

Of the $22 \mathrm{HCC}$ patients studied, $82 \%$ were male and $18 \%$ were female. As mentioned earlier, this tumor is predominant in males, a finding supported by our results. Patients aged 20-45 years were the largest group of individuals and had an earlier age of onset compared to that of a study population in a European study (Romão, 2009), in which 57\% of the patients were older than 70 years, and $43 \%$ were $50-70$ years old.

Owing to the high level of polymorphism of molecules and genes of the main complex and histocompatibility as well as the magnitude of recent advances in typing methods for these markers, the terminology of the system can be highly confusing for non-specialists. In addition, increased understanding of the role of histocompatibility molecules in immune function have added to the complexity of the mechanisms of association between HLA molecules and diseases.

Few studies of the association between histocompatibility antigens and disease in Brazilian populations have been carried out. Available research in Latin American and international databases includes studies of rheumatic fever, pemphigus or endemic pemphigus, chromoblastomycosis, cutaneous leishmaniasis, paracoccidioidomycosis, schistosomiasis, diabetes mellitus, malaria, myasthenia gravis, 21-hydroxylase deficiency, chronic hepatitis, glaucoma, retinal detachment, hereditary angioedema, rheumatoid arthritis, juvenile rheumatoid arthritis, systemic lupus erythematosus, connective tissue disorders, cerebellar ataxia, Chagas disease, Graves' disease, Alport syndrome, acute leukemia, bone marrow failure, and Sydenham's chorea (Donadi, 2000). Our study aimed to explore an association between HCC and HBV carriers and HLA class II.

Given that tumor development is preceded by chronic infection, the immune responses to an infective agent or against tumor antigens may be critical for its development. Various types of HLA molecules have different abilities to connect with and present tumor antigens. We have demonstrated the association of specific HLA alleles susceptible to HBV-related $\mathrm{HCC}$, whereas most previous studies have focused on susceptibility and resistance to chronic hepatitis B (Han et al., 2005). Ramezani et al. (2008) showed that HLA-A*33 is strongly related to susceptibility to hepatitis B persistence and that HLA-DRB*13 is strongly related to protection against hepatitis B persistence in a cross-section of the Iranian population. 
Kummee et al. (2007) studied the association of HLA-DRB1*13 and polymorphism of the tumor necrosis factor (TNF)-alpha gene with clearance of chronic hepatitis B infection and HCC risk in a cross-section of a Thai population. When stratifying chronic HBV patients with and without HCC, allele 863A was significantly more frequent in the HCC group than it was in controls. Haplotype analysis (-863/-308/-238) showed that CGG/CGG homozygosity was a protective marker for HCC. Carriers of genotype 863A have increasing levels of TNF-alpha in liver tissue in response to HBV infection, which can damage liver cells and cause HCC.

Lin et al. (2010) reported 8 control-case studies in an Asian population in a metaanalysis studying HLA-DRB1 alleles and showed that HLA-DRB $1 * 07$ and HLA-DRB $1 * 12$ are significantly associated with HCC risk in the entire population $(\mathrm{P}=0.02$ and $\mathrm{P}=0.02$, respectively). No significant association was shown between the HLA-DRB $1 * 15$ allele and HCC. Subgroup analysis by ethnicity showed that HLA-DRB $1 * 07$, HLA-DRB $1 * 12$, and HLA-DRB $1 * 15$ alleles significantly increased the risk of HCC in Asians $(\mathrm{P}=0.03 ; \mathrm{P}=0.006$, and $\mathrm{P}<0.001$, respectively). Our results showed a significant increase in the frequency of the HLA-DRB1*08 $(\mathrm{P}=0.02)$ allele and significantly lower rates for HLA-DRB1*07 $(\mathrm{P}=0.02)$ compared to those in controls, and low levels of HLA-DRB1* 12 in both groups, suggesting ethnic differences between Asians and Amazonian populations.

Eight control-case studies were included in the final analysis. Of the three HLA-DRB1 alleles studied, HLA-DRB $1 * 07$ and HLA-DRB $1 * 12$ were significantly associated with HCC risk in the entire population [odds ratio $(\mathrm{OR})=1.65,95 \%$ confidence interval $(95 \% \mathrm{CI})=1.08-2.51$, $\mathrm{P}=0.02$ and $\mathrm{OR}=1.59,95 \% \mathrm{CI}=1.09-2.32, \mathrm{P}=0.02$, respectively $]$. No significant association was established for the HLA-DRB $1 * 15$ allele and HCC in the entire population. Subgroup analysis by ethnicity demonstrated that HLA-DRB $1 * 07$, HLA-DRB $1 * 12$, and HLA-DRB $1 * 15$ alleles significantly increased the risk of $\mathrm{HCC}$ in Asians $(\mathrm{OR}=2.10,95 \% \mathrm{CI}=1.06-4.14, \mathrm{P}=0.03 ; \mathrm{OR}=$ $1.73,95 \% \mathrm{CI}=1.17-2.57, \mathrm{P}=0.006$; and $\mathrm{OR}=2.88,95 \% \mathrm{CI}=1.77-4.69, \mathrm{P}<0.001$, respectively).

In 2007, Yang et al. found a high frequency of HLA-DRB $1 * 07$ in HBV patients in northwestern China (17.6\% in HBV patients vs $9.3 \%$ in controls, $\mathrm{P}<0.05)$. Discordance rates in our study showed a low rate of HLA-DRB $1^{*} 07$ in HBV patients compared with that in the control group ( 4.5 vs $20.5 \%, \mathrm{P}=0.02)$.

Zhang et al. (2006) reported that the frequency of HLA-DRB1*12 in patients with persistent HBV was significantly higher than that in a cured Chinese group $(0.230$ vs 0.063 , $\mathrm{P}=0.004)$. In 2003, Amarapurpar et al. pointed out a positive association between HLADRB1*15 and persistent HBV between Indian subjects having HBsAg positive and healthy controls (57.6 vs 25\%). Godkin et al. (2005) showed that HBV clearance in acute infection is associated with vigorous CD4+ T-cell response on the core protein. HLA class II glycoproteins present viral peptides for $\mathrm{CD} 4+\mathrm{T}$ cells and influence immune responses. The affinity bond of the overlay peptide chain, comprising the nucleus protein and HBV envelope, has been measured for HLA glycoproteins encoded by some HLA-DRB1 molecules and compared to published CD4+ peptide-specific cell responses.

Many studies have been conducted using relatively small samples. An insufficient number of individuals may decrease the detection of differences in the distribution of HLA-DRB $1 * 07$, HLA-DRB1*12, and HLA-DRB1*15 alleles between HCC patients and controls, even when a significant difference is present. Studies reporting few associations do not confirm the absence of an association. Many studies have not analyzed a control to the correspondent variables, and conflicting factors are a potential cause of variation in estimates in these studies. Various types of control 
groups could also potentially cause variation in the results of the studies. The effects of interactions of other environmental, behavioral, or viral factors may be unavoidable. The complex interaction between various genes, instead of a single allele, is likely to modulate HCC development. HLA genotype techniques must be considered, because these methodologies differ in important ways that can hamper effective comparisons between studies and influence combined results.

In conclusion, HLA-DRB*08, HLA-DRB3*, and HLA-DQB1*04 are more frequent in HBV and HCC carriers. High alpha-fetoprotein tends to increase this frequency and the frequency of HLA-DRB $1 * 15$. No evidence of important associations between HLA-DBQ1* specificities and disease evolution was found in the population studied. Because serology is a reagent to $\mathrm{HBsAg}$ and total anti-HBc, a higher frequency of HLA-DRB*08 allele was observed.

\section{ACKNOWLEDGMENTS}

We thank the Foundation Supporting Research in the State of Amazonas for assistance, and Jim Hesson of Academic English Solutions for a revision of the English.

\section{REFERENCES}

Amarapurpar DN, Patel ND and Kankonkar SR (2003). HLA class II genotyping in chronic hepatitis B infection. J. Assoc. Physicians India 51: 779-781.

Carrilho FJ, Kikuchi L, Branco F, Gonçalves CS, et al. (2010). Clinical and epidemiological aspects of hepatocellular carcinoma in Brazil. Clinics 65: 1285-1290.

Chávez J, Campana S and Haas P (2003). Panorama da hepatite B no Brasil e no Estado de Santa Catarina. Rev. Panam Salud Publica 14: 91-96.

Donadi EA (2000). How to understand the nomenclature and the mechanisms involved on the association between histocompatibility antigens and alleles with disease. Medicina Ribeirão Preto 33: 7-18.

Ferreira A, Greca D, Tavares E, Moriya Y, et al. (2006). Soroepidemiologia da hepatite B e C em índios Kaingang do Sul do Brasil. Rev. Panam. Salud Publica 20: 230-235.

Ferreira M (2000). Diagnóstico e tratamento da hepatite B. Rev. Soc. Bras. Med. Trop. 33: 389-400.

Godkin A, Davenport M and Hill AV (2005). Molecular analysis of HLA class II associations with hepatitis B virus clearance and vaccine nonresponsiveness. Hepatology 41: 1383-1390.

Gustincich S, Manfioletti G, Del SG, Schneider C, et al. (1991). A fast method for high-quality genomic DNA extraction from whole human blood. Biotechniques 11: 298-300, 302.

Han YN, Yang JL, Zheng SG, Tang G, et al. (2005). Relationship of human leukocyte antigen class II genes with the susceptibility to hepatitis B virus infection and the response to interferon in HBV-infected patients. World $J$. Gastroenterol. 11 (36): 5721-5724.

Kummee P, Tangkijvanich Y, Poovorawan P and Hirankarn N (2007). Association of HLA-DRB1*13 and TNF- $\alpha$ gene polymorphisms with clearance of chronic hepatitis B infection and risk of hepatocellular carcinoma in Thai population. J. Viral Hepat. 14: 841-848.

Lee W (1997). Hepatitis B virus infection. New Engl. J. Med. 337: 1733-1745.

Lin ZH, Xin YN, Dong QJ, Wang Q, et al. (2010). Association between HLA-DRB1 alleles polymorphism and hepatocellular carcinoma a meta-analysis. BMC Gastroenterol. 10: 145.

Miranda E, Moia L, Amaral I, Barbosa M, et al. (2004). Infecções pelos vírus das hepatites B e C e o carcinoma hepatocelular na Amazônia oriental. Rev. Soc. Bras. Med. Trop. 37: 47-51.

Olerup O and Zetterquist H (1992). HLA-DR typing by PCR amplification with sequence-specific primers (PCR-SSP) in 2 hours: an alternative to serological DR typing in clinical practice including donor-recipient matching in cadaveric transplantation. Tissue Antigens 39: 225-235.

Ramezani A, Hasanjani-roshan MR, Kalantar E, Eslamifar A, et al. (2008). Association of human leukocyte antigen polymorphism with outcomes of hepatitis B virus infection. J. Gastroenterol. Hepatol. 23: 1716-1721.

Romão AMM (2009). Relação Entre a Alpha-Fetoproteína, Doença Hepática Crônica e Carcinoma Hepatocelular, e Estudo Imagiológico Tumoral. Master's thesis, Faculdade de Ciências da Saúde, Universidade da Beira Interior, Covilhã. 
Singh R, Kaul R, Kaul A and Khan K (2007). A comparative review of HLA associations with hepatitis B and C viral infections across global populations. World J. Gastroenterol. 13: 1770-1787.

Yang G, Liu J, Han S, Xie H, et al. (2007). Association between hepatitis B virus infection and HLA-DRB1 genotyping in Shaanxi Han patients in northwestern China. Tissue Antigens 69: 170-175.

Zhang SY, Gu HX, Li D, Yang SF, et al. (2006). Association of human leukocyte antigen polymorphism with hepatitis B virus infection and genotypes. Jpn. J. Infect. Dis. 59: 353-357. 